\title{
ITINERARIOS EDITORIALES. RECORRIDOS POSIBLES PARA LA CONFORMACIÓN DE UNA COLECCIÓN DE COMUNICACIÓN
}

\author{
BRUNO DE ANGELIS \\ UNIVERSIDAD NACIONAL DE QUILMES \\ ARGENTINA \\ BDEANGELIS@UNQ.EDU.AR
}

HTTP://DX.DOI.ORG/10.5902/2316882X23139 
ITINERÁRIOS EDITORIAIS. AS POSSÍVEIS VIAS PARA A FORMAÇÃO DE UMA COLEÇÃO DE COMUNICAÇÃO

Resumo: Baseado no trabalho anterior, descrevendo o surgimento da coleção Comunicación de masa, Editorial Siglo XXI-Argentina, este documento vai investigar o conjunto de circunstâncias políticas, disciplinar e de publicação que serviu como pano de fundo para uma das primeiras coleções de comunicação da Argentina.

Palavras chave: Editoras; Comunicação; Coleções

ITINERARIOS EDITORIALES. RECORRIDOS POSIBLES PARA LA CONFORMACIÓN DE UNA COLECCIÓN DE COMUNICACIÓN

Resumen: Con base en trabajos anteriores, que describen la emergencia de la colección Comunicación de masa, de la editorial Siglo XXI-Argentina, el presente trabajo se ocupará de indagar en el conjunto de circunstancias políticas, disciplinares y del mundo editorial que funcionaron como antecedentes de una de las primeras colecciones de Comunicación de Argentina.

Palabras clave: Editoriales; Comunicación; Colecciones

EDITORIAL ITINERARIES. POSSIBLE ROUTES FOR THE FORMATION OF A COLLECTION OF COMMUNICATION

Abstract: Based on previous work, describing the emergency of the collection Comunicación de masa, published by Editorial Siglo XXI-Argentina, this paper will address the investigation of political contexts, disciplinary and publishing that served as background of one of the first collections of Communication within Argentina.

Keywords: Publishers; Communication; Collections 


\section{INTRODUCCIÓN}

En un ponencia anterior, presentada en ALAIC 2012, en Montevideo, hicimos referencia a la generación de colecciones editoriales, a la relación autor, editor y director de colección, a lo que podría significar la puesta en serie de un ejemplar, a todo lo que ello implica al momento de la toma de esa decisión y quién en esa cadena de relaciones toma las decisiones. La "puesta en serie", decíamos, la inclusión en una colección implica un ordenamiento, una clasificación, regida por un criterio que proviene inicialmente del editor y que, una vez instalado el sistema, podrá asignarse a una nueva figura, como es el director de colección. Incorporar una obra dentro de una colección va a constituir una estrategia, que en algunos casos otorgará una nueva identidad al texto, al destinarlo, por ejemplo, a un público a veces impensado por el autor. (De Angelis, 2012).

En ese artículo dimos cuenta de la Colección Comunicación de masa, dirigida por Héctor Schmucler, que se publicó entre 1972 y 1973 en la editorial Siglo XXI editores de Argentina. La colección constaba de siete volúmenes:

- 1972: Para leer al pato Donald. Comunicación de masa y colonialismo, de Ariel Dorfman y Armand Mattelart. Con ese ejemplar -publicado ese mismo año previamente por Ediciones Universitarias de Valparaíso, en Chile- se inicia la colección (a finales de 1973 ya se publicaba la $7^{\circ}$ edición).

- 1972: Cine, Cultura y Descolonización, de Fernando Solanas y Octavio Getino. Primera edición.

- 1973: El lenguaje de la publicidad, de Luisa Block de Behar. Primera edición.

- 1973: La información de clase, de Vladimir Ilich Lenin, traducción de Augusto Bianco (introducción) y Marisa Cortazzo (textos). Primera edición. La primera edición en italiano se publicó en 1972 por Guaraldi Editor, Rimini (L'informazione di classe).

- 1973: La comunicación masiva en el proceso de liberación, de Armand Mattelart. Primera edición.

- 1973: Agresión desde el espacio. Cultura y napalm en la era de los satélites, de Armand Mattelart. Segunda edición.

- 1973: El cine como propaganda política, de Alexander Medvedkin. Primera edición. 
En este trabajo nos interesa indagar en los antecedentes editoriales de la colección y del grupo de editores que permitieron que surgiera, por un lado; y por otro, en los antecedentes políticos y disciplinares que colaboraron en su emergencia.

Con la ventaja que nos permite la mirada a distancia de los hechos podemos decir que esta colección se conformó a partir de tres antecedentes sin los cuales, tal vez no hubiera sido posible. Uno de ellos relacionado con la experiencia de Armand Mattelart en Latinoamérica y su vinculación con quien sería informalmente su editor en Argentina: Héctor Schmucler. Otro, tiene que ver con la editorial Ediciones Signos y su posterior fusión con Siglo XXI Argentina. Y un tercer antecedente lo constituye la existencia previa de dos volúmenes, que por su contenido, autoría y contexto de publicación fueron volúmenes posibles de la colección, aunque nunca formaron parte de ella.

A partir de estos tres recorridos intentaremos reseñar los momentos previos a la conformación de Comunicación de masa.

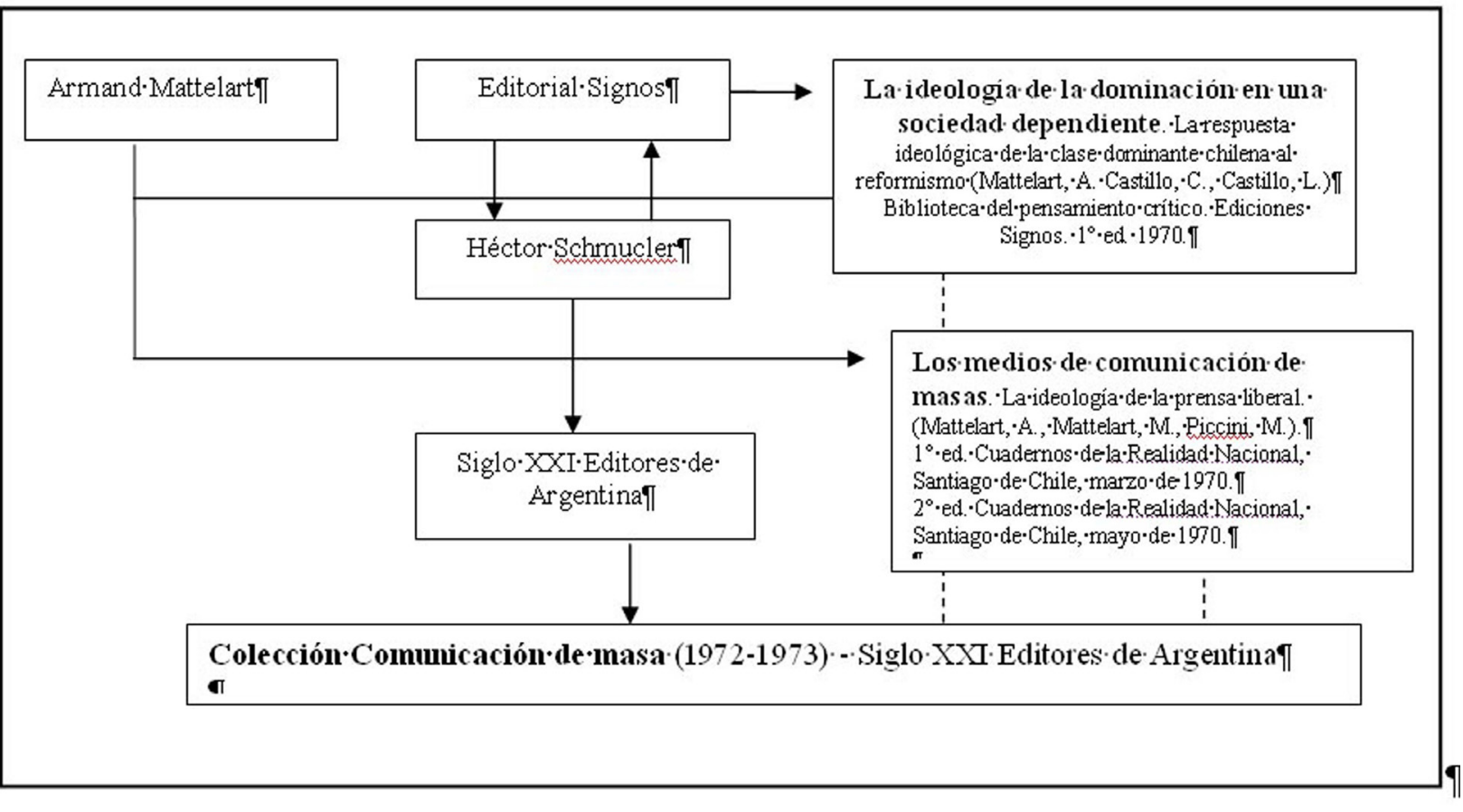

\section{HÉCTOR SCHMUCLER Y EDICIONES SIGNOS}

Héctor Schmucler viajó a Francia en 1965 para estudiar con Roland Barthes, en ese momento en Francia se produce todo un giro en la preocupación por las formas masivas de comunicación con una fuerte impronta semiológica (el enfoque semiológico se desarrolla en Argentina fundamentalmente con Eliseo Verón, a fines de la década de 1960, también

Rev.Cad.Comun. Santa Maria, v.21, n.2, art 3, p. 56 de 65, maio/ago.2017 
Armand Mattelart parte del enfoque semiológico, aunque ya bajo la corriente crítica que se desarrollaba en Chile).

Regresó a la Argentina a fines de 1968 y en 1969 funda la revista Los libros. Por sus antecedentes en semiología lo invitan a dar un curso en la Escuela de periodismo de La Plata, y crean la materia Semiología de la prensa escrita:

\footnotetext{
"lo semiológico fue luego el gran elemento innovador de las carreras de comunicación, [...] entonces, lo semiótico aparecía como disruptivo pero ;ojo! era lo semiótico a fines del 60 , en el medio latinoamericano, estaba todo cargado de un interés, de una preocupación histórico-política, no político partidario como a veces se proclamaba, no, lo político que fue creciendo como elemento, como parte de un proceso de aspiración a la transformación radical de la sociedad". (cita: entrevista personal 2012)
}

Algunos años más adelante, en 1973, Schmucler junto con Nicolás Casullo y Aníbal Ford dan un seminario en la Facultad de Filosofía y Letras de la UBA -"en medio del proceso de transformación político cultural de la facultad"- Ilamado Comunicación masiva.

Entre todas las actividades que desarrollaba Schmucler, a su regreso de Francia, formó parte de la breve vida de la editorial Signos.

Los historiadores Enrique Tándeter y Juan Carlos Garavaglia fueron los generadores de este emprendimiento editorial que se focalizó en libros de historia y ciencias sociales. Tándeter y Garavaglia, al finalizar sus carreras universitarias se dedicaron a tantear diversas posibilidades en el mundo editorial, desde Jorge Álvarez, pasando por Daniel Divinski (de la Flor) y Galerna, dirigida por Jorge Schavelzon. También contactaron con Orfila Reynal (Siglo XXI) y finalmente entraron en contacto con la Editorial Pasado y Presente (José Aricó y Héctor Schmucler, entre otros) y surgió Ediciones Signos.

Fue una editorial que funcionó aproximadamente desde comienzos de 1970 a mediados de 1971. Formó un catálogo de alrededor de veinte libros relativos a ciencias sociales, literatura y política. La editorial deja de existir en el momento de la apertura de Siglo XXI editores de Argentina.

La organización de Signos no fue muy estricta, se organizó a partir de una división básica de funciones (Enrique Tándeter como gerente, José Aricó y Juan Carlos Garavaglia en la tarea estrictamente editorial y Héctor Schmucler se ocupaba de la difusión). Si bien las decisiones editoriales las

Rev.Cad.Comun. Santa Maria, v.21, n.2, art 3, p. 57 de 65, maio/ago.2017 
tomaba el núcleo original de manera conjunta, podemos reconocer la presencia de sus miembros en cada una de las colecciones.

Se distinguen nítidamente las colecciones que forman parte de la editorial de aquellas que ésta distribuye: los "Cuadernos de Pasado y Presente" y "Ediciones de PyP”. Ambas colecciones tiene una existencia previa, y por lo tanto autónoma de Signos.

La colección "Rhesis" era la única en la que figuraba el nombre de su director en los libros, probablemente tratando de trasladar a la colección y la editorial el reconocimiento que tenía en ese momento Héctor Schmucler como director de la revista Los libros y por su relación con Roland Barthes.

Signos publica en su colección Economía y Sociedad el libro de Mattelart, Castillo y Castillo, La ideología de la dominación en una sociedad dependiente, en noviembre de 1970, Mattelart llega a la editorial por recomendación de Carlos Assadourian, (que junto a Mabel Piccini, quien fue su colaboradora, distribuían los Cuadernos de Pasado y Presente en Chile).

Este fue el comienzo de la relación profesional entre Mattelart y Schmucler que generará el inicio de sus investigaciones en proyectos relacionados con el hasta entonces incipiente campo de estudios de la comunicación masiva.

De todos los proyectos conjuntos entre Schmucler y Mattelart quizás el más reconocido fue la revista Comunicación y Cultura que ofició de soporte a la consolidación del campo de la comunicación.

A mediados de 1971, como adelantamos, Signos se disuelve luego de acordar la apertura de Siglo XXI-Argentina, en esa fusión, la nueva editorial dividió las tareas de manera tal que el grupo que dirigía Signos se ocuparía de las prácticas de edición literaria, mientras que los relacionados con Siglo XXI dirigirían las tareas administrativas. En los años siguientes Siglo XXI publicó la mayoría de los títulos que proyectaba concretar Signos. (Diego García, 2012, Signos).

1 Diego García (2012) interpreta que el nombre de I acolección muestra claramente su origen académico y un llamado a la nueva crítica y que en relación con los libros publicados se pretende "científico y de vanguardia": "El nombre de la colección remitía a un concepto técnico de la crítica y la historia de la literatura: un discurso o recitado en tono épico realizado, habitualmente, por un mensajero, cuyo contenido son las acciones que tienen lugar fuera del escenario".

Rev.Cad.Comun. Santa Maria, v.21, n.2, art 3, p. 58 de 65, maio/ago.2017 


\section{ARMAND MATTELART EN CHILE}

A partir del itinerario intelectual de Armand Mattelart que trazó Mariano Zarowsky (2013), resumiremos acá su recorrido por Chile que inició en 1962 como docente en la Pontificia Universidad Católica de Santiago de Chile.

Zarowsky relata que "La crisis del proyecto conducido por la Democracia Cristiana de Eduardo Frei (1964-1970) y los efectos en el mundo intelectual de la reforma universitaria que tuvo lugar en las universidades del país andino entre 1967 y 1969 (más puntualmente, la creación en la Universidad Católica del Centro de Estudios de la Realidad Nacional, el CEREN, del cual fue uno de sus fundadores, enmarcaron un giro en su itinerario que fue simultáneamente disciplinar -hacia los estudios de comunicación-, teórico-epistemológico -hacia el marxismo-y político -hacia la llamada 'nueva izquierda"”. (Zarowsky, 2013: 23)

Durante el gobierno de Salvador Allende, las ciencias sociales chilenas tuvieron un grado importante de desarrollo, institucionalización y autonomización. Desde las fuerzas de izquierda se planteaba crear una "'nueva cultura' que contribuyera a forjar una transición socialista en el marco de un aparato cultural y comunicacional que permanecía en buena medida en manos de las clases tradicionales [... ] que ensayaban nuevas estrategias de desestabilización y contrainsurgencia que se sostenían más en el manejo de la comunicación y la información que en la fuerza militar”. (Zarowsky, 2013: 24)

El autor de Del laboratorio chileno a la comunicación-mundo, destaca dos aspectos fundamentales para entender el origen del pensamiento de Mattelart y su contribución a la reflexión sobre la comunicación: por un lado el desarrollo de las ciencias sociales en Chile y por otro la "centralidad del debate sobre la cultura y la comunicación". (Ibidem)

En poco tiempo, Mattelart se erigió como un referente intelectual para un sector de la nueva izquierda, "que exploraba entonces alternativas políticas, sociales y culturales” (Ibidem). Y a su vez se convertiría en uno de los principales referentes de los estudios de comunicación en Latinoamérica. En ese sentido, Mirta Varela ubica a Mattelart como una de las principales referencias de la emergencia de los estudios de comunicación de fines de la década de 1960 y luego durante la de 1970. Destaca que su recorrido fue fundamental para la concreción de un espacio de investigación

Rev.Cad.Comun. Santa Maria, v.21, n.2, art 3, p. 59 de 65, maio/ago.2017 
"cuyo objetivo inmediato fue la denuncia del modo en que las empresas, la tecnología y los mensajes norteamericanos invadían América Latina". (Varela, 2010)

En su proceso, Mattelart va formando redes internacionales en Latinoamérica, como vimos en un apartado anterior se conecta con la gente de Cuadernos de Pasado y Presente, luego es invitado a participar en un número especial sobre Chile para la revista Los Libros (1971) y se consolida su relación intelectual con Schmucler.

En 1973 se inicia la colección Comunicación de masa con la publicación en Siglo XXI de Para leer al Pato Donald. Así multiplicaba su circulación por toda Sudamérica y contribuía a la consolidación disciplinar de Mattelart en el campo de la comunicación.

\section{LOS VOLÚMENES PREVIOS}

Schmucler destaca el papel de Mattelart como "mediador" y "promotor de singulares cruces de pensamientos múltiples" y por ello considera que no es casual que muchos de sus libros aparezcan en colaboración, con una fuerte impronta propia, pero siempre estimulando el pensar del otro. En esa línea se inscriben los dos volúmenes a los que hacíamos referencia. En marzo de 1970, el CEREN (Centro de Estudios de la Realidad Nacional) de la Universidad Católica de Chile publica como número especial de la revista Cuadernos de la Realidad Nacional el libro Los medios de comunicación de masas. La ideología de la prensa liberal de Armand Mattelart, Mabel Piccini, Michéle Mattelart. En la página inicial como advertencia al lector se lee: "La coyuntura en que apareció este libro, así como la inspiración de los trabajos que lo componen, le aseguró una trayectoria muy polémica en el intenso debate que vivía Chile. Como primera etapa en el país de una aproximación crítica a los medios de comunicación, de esta manera abrió nuevas perspectivas no sólo en el dominio académico de las Ciencias Sociales sino en el debate ideológico global. En la batalla electoral de los meses de junio-agosto de 1970, fue tratado de obra de 'marxismo ficción' por los representantes de la oligarquía tradicional y apreciado como documento esclarecedor por el candidato de la Unidad Popular, Salvador Allende".

Sobre el otro volumen ya hicimos una breve referencia: en noviembre de 1970 Ediciones Signos publica La ideología de la dominación en una so-

Rev.Cad.Comun. Santa Maria, v.21, n.2, art 3, p. 60 de 65, maio/ago.2017 
ciedad dependiente. La respuesta ideológica de la clase dominante chilena al reformismo, de Mattelart en coautoría con Carmen Castillo y Leonardo Castillo. Este libro no estaba planificado por la editorial ${ }^{2}$ sino que fue producto de la urgencia política de sus autores (quienes financiaron la publicación) para que ese libro circulara antes de la asunción de Salvador Allende. El propósito que empujó a su publicación radica en poner en evidencia "la ideología que dota de coherencia al proyecto de dominación social de una clase en una coyuntura y una dinámica específicas". La coyuntura a la que se refiere corresponde a la sociedad chilena en el momento de aplicación de una reforma agraria, que auspiciada por los sectores medios tiende a descompaginar la racionalidad de la dominación de los grupos tradicionales.

Los autores advierten que este estudio no puede ser tomado como un fenómeno aislado, sino que se "inscribe en una línea heurística que desde hace algún tiempo sirve de eje a nuestras observaciones". Incluyen en esta línea "la investigación que junto con Michéle Mattelart y Mabel Piccini realizamos en el curso del año 1969 sobre la ideología de la prensa liberal". (Cuadernos de la Realidad Nacional, Santiago $\mathrm{N}^{\circ} 3$ )

Mattelart ubica a esta primera publicación como el "primer peldaño" donde pudieron aproximarse a un estudio crítico de los mensajes emitidos por "los ideólogos de una clase dominante dependiente" a través de la prensa manipulada por dicha clase. En ese caso trabajaron con el diario de mayor circulación, las revistas de ídolos y la prensa "seudoamorosa".

En el caso de la Ideología de la dominación... "también calibramos la estructura de poder de la información comprobando que la estructura de dominación sobre los medios de comunicación de masas corresponde plenamente a la estructura implícita de los mensajes que transmiten. La mitología vehiculizada por estos medios es funcional al sistema de poder y asegura una continua reproducción de su legitimidad" (Mattelart et al, 1970:5)

Mattelart incluye estos trabajos (y podríamos pensar que los que siguen) en "una corriente de trabajos inaugurados en América Latina por Eliseo Verón ${ }^{3}$ y sus colaboradores. Corriente eminentemente crítica, [... ]"

2 Ver Plan de publicaciones de Ediciones Signos en García, D. (2012).

3 Referencia interesante de la pertenencia a una corriente compartida con E. Rev.Cad.Comun. Santa Maria, v.21, n.2, art 3, p. 61 de 65, maio/ago.2017 
que "Suscribe la necesidad de despejar la ilusión objetiva -que el empirismo ha sacralizado como objetividad suprema- para que emerjan las estructuras ocultas o los significados segundos que hacen inteligibles los mensajes funcionales de la dominación social”. (Ibidem)

\section{CONCLUSIÓN}

El uso de fórmulas de denuncia, lo coyuntural del contenido y la publicación de textos urgentes hablan de ediciones marcadas por el ritmo de la política. La crítica tenía un sentido político claro y una intención de intervenir explícitamente buscando desmantelar los discursos construidos por los poderes establecidos. La política como dadora de sentido a todas las demás prácticas nos obliga a incluir aquí a la actividad editorial, como forma de intervención política. En este caso, trasciende la casa editorial de origen, es más bien un proyecto de intervención política que incluye una producción editorial urgente que primero se publica en Chile a instancias del CEREN en Cuadernos de la Realidad Nacional, luego en Ediciones Signos (en la Colección Economía y sociedad) y finalmente toma forma de colección en Comunicación de masa, en Siglo XXI Editores, intentando completar con cada "peldaño", una serie que fuera por encima de la editorial que circunstancialmente la publicara.

Este proyecto editorial, indispensable como accionar político, y que es impensable sin la figura de Mattelart y Schmucler finaliza, en lo que respecta a la colección, en 1973, coincidiendo con el golpe en Chile y el regreso de Mattelart a Europa. Seguirán la revista Comunicación y cultura con sedes en diferentes países y otros proyectos en conjunto.

Del plan inicial de ediciones de Signos se publicaron alrededor de veinte volúmenes, algunos como el de Mattelart no estaban en el plan inicial, parte de los demás fueron publicados por Siglo XXI luego de la fusión. Entre otras cosas, Siglo XXI heredó la impronta de Mattelart a través de Héctor Schmucler que continuó el proceso iniciado en Chile por Mattelart y su propio proceso y concretó en una colección que colaboró a la consolidación de una línea de investigación de los incipientes estudios de comunicación.

Verón que relativiza quizás la importancia de la posterior polémica entre Lenguajes y Comunicación y Cultura.

Rev.Cad.Comun. Santa Maria, v.21, n.2, art 3, p. 62 de 65, maio/ago.2017 


\section{REFERENCIAS}

CASULLO, N. (1996). "Diálogo con los investigadores argentinos de la comunicación/ cultura/medios”. Buenos Aires: Revista Mapa Nocturno.

DE ANGELIS, B. (2012). "Colecciones editoriales de la década de 1960 y principios de 1970 en Argentina. Las colecciones sobre comunicación” Dossiê Temático: Produção Editorial. Revista Cadernos de Comunicação. ISSN 1677-9061, v. 16, n. 1 (Jan-Jun). Disponible en: <http://cascavel.ufsm.br/revistas/ojs2.2.2/index.php/ccomunicacao/article/ view/5837>

DE DIEGO, J. (dir.) (2006). Editores y políticas editoriales en Argentina (1880-2000). Buenos Aires: FCE.

DE SAGASTIZABAL, L. (1995). La edición de libros en la Argentina. Una empresa de cultura. Buenos Aires: Eudeba.

GARCÍA, D. (2012). "Signos", en: Políticas de la Memoria, Buenos Aires: CEDINCI, N 10, $11 / 12$.

LENARDUZZI, V. (1999). Comunicación y cultura. Itinerarios, ideas y pasiones. Buenos Aires: Eudeba.

Más libros para más. Colecciones del Centro Editor de América Latina (2008). Buenos Aires: Ediciones Biblioteca Nacional, Serie Índices y Bibliografías; edición de Judith Gociol et al.

SCHMUCLER, H. (1997). Memoria de la comunicación. Buenos Aires: Editorial Biblos.

MATTELART, A. et al (1970). La ideología de la dominación en una sociedad dependiente. La respuesta ideológica de la clase dominante chilena al reformismo. Buenos Aires: Ediciones Signos.

MATTELART, A. et al (1976). Los medios de comunicación de masas. La ideología de la prensa liberal. Buenos Aires: Ediciones Signos.

NEIBURG, F. y M. PLOTKING (compiladores) (2004). Intelectuales y Expertos. La Constitución del Conocimiento Social en la Argentina. Buenos Aires: Paidós.

Rev.Cad.Comun. Santa Maria, v.21, n.2, art 3, p. 63 de 65, maio/ago.2017 
CADERNOS DE COMUNICAÇÃO

UNIVERSIDADE FEDERAL DE SANTA MARIA

OLIVERO, I. (1999). L'invention de la Collection. De la diffusion de la littérature et des savoirs à la formation du citoyen au XIXe siècle. París: Éditions de l'IMEC / Éditions de la MSH.

RIVERA, J. (1987). La investigación en comunicación social en Argentina. Buenos Aires: Ed. Punto Sur.

ALFONSO, A. et al (comps.). 70 años de Periodismo y Comunicación en América Latina. Memoria y perspectivas. La Plata: Universidad Nacional de La Plata.

VARELA, M. (2010), “Intelectuales y medios de comunicación”. En Carlos Altamirano. Historia de los intelectuales en América Latina II. Los avatares de la "ciudad letrada" en el siglo XX, Buenos Aires, Katz.

ZAROWSKY, M. (2013). Del laboratorio chileno a la comunicación-mundo. Un itinerario intelectual de Armand Mattelart, Buenos Aires: Colección Comunicación, Medios Cultura, Editorial Biblos. 


\section{Bruno De Angelis}

Docente investigador del Departamento de Ciencias So-
ciales de la Universidad Nacional de Quilmes. Director de
Materiales Didácticos de la Secretaría de Educación Virtual
(UNQ). Co-director de la Colección Serie Digital, Editorial
de la Universidad Nacional de Quilmes. Lic. en Comunicaci-
ón Social.
E-mail: bdeangelis@unq.edu.ar

RECEBIDO EM: $18 / 07 / 2016$

ACEITO EM: 16/09/2016

Rev.Cad.Comun. Santa Maria, v.21, n.2, art 3, p. 65 de 65, maio/ago.2017 